\title{
Ratio-Cum-Product Estimators of Population Mean Using Known Population Parameters of Auxiliary Variates
}

\author{
Rajesh Tailor ${ }^{a}$, Rajesh Parmar ${ }^{a}$, Jong-Min Kim $^{1, b}$, Ritesh Tailor ${ }^{c}$ \\ ${ }^{a}$ School of Studies in Statistics, Vikram University \\ ${ }^{b}$ Statistics, Division of Science and Mathematics, University of Minnesota-Morris \\ ${ }^{c}$ Institute of Would Science and Technology
}

\begin{abstract}
This paper suggests two ratio-cum-product estimators of finite population mean using known coefficient of variation and co-efficient of kurtosis of auxiliary characters. The bias and mean squared error of the proposed estimators with large sample approximation are derived. It has been shown that the estimators suggested by Upadhyaya and Singh (1999) are particular case of the suggested estimators. Almost ratio-cum product estimators of suggested estimators have also been obtained using Jackknife technique given by Quenouille (1956). An empirical study is also carried out to demonstrate the performance of the suggested estimators.
\end{abstract}

Keywords: Ratio-cum-product estimator, population mean, coefficient of variation, coefficient of kurtosis, bias, mean squared error.

\section{Introduction}

Use of auxiliary information has been in practice to increase the efficiency of the estimators. When the population mean of an auxiliary variate is known, so many estimators for population parameter $(s)$ of study variate have been discussed in the literature. When correlation between study variate and auxiliary variate is positive (high) ratio method of estimation (Cochran, 1940) is used. On the other hand if the correlation is negative, product method of estimation (Robson, 1957; Murthy, 1967) is preferred. In practice information on coefficient of variation $(\mathrm{CV})$ of an auxiliary variate is seldom known. Sisodia and Dwivedi (1981) suggested a modified ratio estimator for population mean of the study variate. Later on Upadhyaya and Singh (1999), derived another ratio and product type estimators using coefficient of variation and coefficient of kurtosis of the auxiliary variate. Singh (1967) utilized information on two auxiliary variates $x_{1}$ and $x_{2}$ and suggested a ratio-cum-product estimator for population mean. Singh and Tailor (2005) utilized known correlation coefficient between auxiliary variates $\left(\rho_{x_{1} x_{2}}\right) x_{1}$ and $x_{2}$.

Singh and Tailor (2005) motivates authors to suggest ratio-cum-product estimators of population mean utilizing the information on co-efficient of variation of auxiliary variates i.e. $C_{x_{1}}$ and $C_{x_{2}}$ and co-efficient of kurtosis of auxiliary variates $\beta_{2}\left(x_{1}\right)$ and $\beta_{2}\left(x_{2}\right)$ besides the population means $\left(\bar{X}_{1}\right.$ and $\bar{X}_{2}$ ) of auxiliary variates $x_{1}$ and $x_{2}$.

Let $U=\left\{U_{1}, U_{2}, \ldots, U_{N}\right\}$ be a finite population of $N$ units. Suppose two auxiliary variates $x_{1}$ and $x_{2}$ are observed on $U_{i}(i=1,2, \ldots, N)$, where $x_{1}$ is positively and $x_{2}$ is negatively correlated with the study variate $y$. A simple random sample of size $n$ with $n<N$, is drawn using simple random sampling without replacement(SRSWOR) from the population $U$ to estimate the population mean $(\bar{Y})$

\footnotetext{
${ }^{1}$ Corresponding author: Associate Professor, Statistics, Division of Science and Mathematics, University of MinnesotaMorris, MN 5627, USA. E-mail: jongmink@morris.umn.edu
} 
of study character $y$, when the population means $\bar{X}_{1}=\sum_{i=1}^{N} x_{1 i} / N$ and $\bar{X}_{2}=\sum_{i=1}^{N} x_{2 i} / N$ of $x_{1}$ and $x_{2}$ respectively are known.

Usual ratio and product estimators given by Cochran (1940) and Robson (1957) respectively for estimating the population mean $\bar{Y}$ respectively are defined as

$$
\begin{aligned}
& \bar{y}_{R}=\bar{y}\left(\frac{\bar{X}_{1}}{\bar{x}_{1}}\right), \\
& \bar{y}_{P}=\bar{y}\left(\frac{\bar{x}_{2}}{\bar{X}_{2}}\right) .
\end{aligned}
$$

Utilizing the information on co-efficient of variations $\left(C_{x_{1}}\right.$ and $\left.C_{x_{2}}\right)$ and co-efficient of kurtosis $\left(\beta_{2}\left(x_{1}\right)\right.$ and $\left.\beta_{2}\left(x_{2}\right)\right)$, Upadhyaya and Singh (1999) suggested ratio and product estimators as

$$
\begin{aligned}
& \hat{\bar{Y}}_{1}=\bar{y}\left(\frac{\bar{X}_{1} C_{x_{1}}+\beta_{2}\left(x_{1}\right)}{\bar{x}_{1} C_{x_{1}}+\beta_{2}\left(x_{1}\right)}\right), \\
& \hat{\bar{Y}}_{2}=\bar{y}\left(\frac{\bar{x}_{2} C_{x_{2}}+\beta_{2}\left(x_{2}\right)}{\bar{X}_{2} C_{x_{2}}+\beta_{2}\left(x_{2}\right)}\right), \\
& \hat{\bar{Y}}_{3}=\bar{y}\left(\frac{\bar{X}_{1} \beta_{2}\left(x_{1}\right)+C_{x_{1}}}{\bar{x}_{1} \beta_{2}\left(x_{1}\right)+C_{x_{1}}}\right), \\
& \hat{\bar{Y}}_{4}=\bar{y}\left(\frac{\bar{x}_{2} \beta_{2}\left(x_{2}\right)+C_{x_{2}}}{\bar{X}_{2} \beta_{2}\left(x_{2}\right)+C_{x_{2}}}\right) .
\end{aligned}
$$

To estimate $\bar{Y}$, Singh (1967) suggested a ratio-cum-product estimator as

$$
\hat{\bar{Y}}_{5}=\bar{y}\left(\frac{\bar{X}_{1}}{\bar{x}_{1}}\right)\left(\frac{\bar{x}_{2}}{\bar{X}_{2}}\right) .
$$

Assuming that the correlation coefficient $\left(\rho_{x_{1} x_{2}}\right)$ between auxiliary characters $x_{1}$ and $x_{2}$ is known, Singh and Tailor (2005) suggested a ratio-cum-product estimator of $\bar{Y}$

$$
\hat{\bar{Y}}_{6}=\bar{y}\left(\frac{\bar{X}_{1}+\rho_{x_{1} x_{2}}}{\bar{x}_{1}+\rho_{x_{1} x_{2}}}\right)\left(\frac{\bar{x}_{2}+\rho_{x_{1} x_{2}}}{\bar{X}_{2}+\rho_{x_{1} x_{2}}}\right) .
$$

To the first degree of approximation the mean squared error(MSE) of the estimators $\bar{y}_{R}, \bar{y}_{P}, \hat{\bar{Y}}_{1}, \hat{\bar{Y}}_{2}, \hat{\bar{Y}}_{3}$, $\hat{\bar{Y}}_{4}, \hat{\bar{Y}}_{5}$ and $\hat{\bar{Y}}_{6}$ respectively are

$$
\begin{aligned}
& \operatorname{MSE}\left(\bar{y}_{R}\right)=\theta \bar{Y}^{2}\left[C_{y}^{2}+C_{x_{1}}^{2}-2 \rho_{y x_{1}} C_{y} C_{x_{1}}\right], \\
& \operatorname{MSE}\left(\bar{y}_{P}\right)=\theta \bar{Y}^{2}\left[C_{y}^{2}+C_{x_{2}}^{2}+2 \rho_{y x_{2}} C_{y} C_{x_{2}}\right], \\
& \operatorname{MSE}\left(\hat{\bar{Y}}_{1}\right)=\theta \bar{Y}^{2}\left[C_{y}^{2}+\lambda_{1} C_{x_{1}}^{2}-2 \rho_{y x_{1}} \lambda_{1} C_{y} C_{x_{1}}\right], \\
& \operatorname{MSE}\left(\hat{\bar{Y}}_{2}\right)=\theta \bar{Y}^{2}\left[C_{y}^{2}+\lambda_{2} C_{x_{2}}^{2}+2 \rho_{y x_{2}} \lambda_{2} C_{y} C_{x_{2}}\right], \\
& \operatorname{MSE}\left(\hat{\bar{Y}}_{3}\right)=\theta \bar{Y}^{2}\left[C_{y}^{2}+\gamma_{1}^{2} C_{x_{1}}^{2}-2 \rho_{y x_{1}} \gamma_{1} C_{y} C_{x_{1}}\right], \\
& \operatorname{MSE}\left(\hat{\bar{Y}}_{4}\right)=\theta \bar{Y}^{2}\left[C_{y}^{2}+\gamma_{2}^{2} C_{x_{2}}^{2}+2 \rho_{y x_{2}} \gamma_{2} C_{y} C_{x_{2}}\right], \\
& \operatorname{MSE}\left(\hat{\bar{Y}}_{5}\right)=\theta \bar{Y}^{2}\left[C_{y}^{2}+C_{x_{1}}^{2}\left(1-2 K_{y x_{1}}\right)+C_{x_{2}}^{2}\left\{1+2\left(K_{y x_{2}}-K_{x_{1} x_{2}}\right)\right\}\right]
\end{aligned}
$$


and

$$
\operatorname{MSE}\left(\hat{\bar{Y}}_{6}\right)=\theta \bar{Y}^{2}\left[C_{y}^{2}+\mu_{1}^{*} C_{x_{1}}^{2}\left(\mu_{1}^{*}-2 K_{y x_{1}}\right)+\mu_{2}^{*} C_{x_{2}}^{2}\left\{\mu_{2}^{*}+2\left(K_{y x_{2}}-\mu_{1}^{*} K_{x_{1} x_{2}}\right)\right\}\right],
$$

where

$$
\begin{aligned}
& K_{y x_{1}}=\rho_{y x_{1}}\left(\frac{C_{y}}{C_{x_{1}}}\right), \quad K_{y x_{2}}=\rho_{y x_{2}}\left(\frac{C_{y}}{C_{x_{2}}}\right), \quad K_{x_{1} x_{2}}=\rho_{x_{1} x_{2}}\left(\frac{C_{x_{1}}}{C_{x_{2}}}\right), \quad C_{y}=\frac{S_{y}}{\bar{Y}}, \quad \lambda_{i}=\frac{\bar{X}_{i} C_{x_{i}}}{\bar{X}_{i} C_{x_{i}}+\beta_{2}\left(x_{i}\right)}, \\
& \gamma_{i}=\frac{\bar{X}_{i} \beta_{2}\left(x_{i}\right)}{\bar{X}_{i} \beta_{2}\left(x_{i}\right)+C_{x_{i}}}, \quad \mu_{1}^{*}=\frac{\bar{X}_{i}}{\bar{X}_{i}+\rho_{x_{1} x_{2}}}, \quad \theta=\left(\frac{1}{n}-\frac{1}{N}\right), \quad C_{x_{i}}=\frac{S_{x_{i}}}{\bar{X}_{i}}, \quad \rho_{y x_{i}}=\frac{S_{y x_{i}}}{S_{y} S_{x_{1}}}, \\
& S_{y}^{2}=\frac{\sum_{j=1}^{N}\left(y_{j}-\bar{Y}\right)^{2}}{N-1}, \quad S_{x_{i}}^{2}=\frac{\sum_{j=1}^{N}\left(x_{i j}-\bar{X}_{i}\right)^{2}}{N-1} \quad \text { and } \quad S_{y x_{i}}^{2}=\frac{\sum_{j=1}^{N}\left(y_{j}-\bar{Y}\right)\left(x_{i j}-\bar{X}_{i}\right)}{N-1},
\end{aligned}
$$

where $(i=1,2)$.

\section{Proposed Estimator}

Assuming that the information on coefficient of variation $\left(C_{x_{1}}\right.$ and $\left.C_{x_{2}}\right)$ and co-efficient of kurtosis $\left(\beta_{2}\left(x_{1}\right)\right.$ and $\left.\beta_{2}\left(x_{2}\right)\right)$ of auxiliary variate $x_{1}$ and $x_{2}$, are known, the proposed estimators are

$$
\begin{aligned}
& \hat{\bar{Y}}_{7}=\bar{y}\left(\frac{\bar{X}_{1} C_{x_{1}}+\beta_{2}\left(x_{1}\right)}{\bar{x}_{1} C_{x_{1}}+\beta_{2}\left(x_{1}\right)}\right)\left(\frac{\bar{x}_{2} C_{x_{2}}+\beta_{2}\left(x_{2}\right)}{\bar{X}_{2} C_{x_{2}}+\beta_{2}\left(x_{2}\right)}\right), \\
& \hat{\bar{Y}}_{8}=\bar{y}\left(\frac{\bar{X}_{1} \beta_{2}\left(x_{1}\right)+C_{x_{1}}}{\bar{x}_{1} \beta_{2}\left(x_{1}\right)+C_{x_{1}}}\right)\left(\frac{\bar{x}_{2} \beta_{2}\left(x_{2}\right)+C_{x_{2}}}{\bar{X}_{2} \beta_{2}\left(x_{2}\right)+C_{x_{2}}}\right) .
\end{aligned}
$$

To obtain the bias and mean squared error of the proposed estimators, we assume that $\bar{y}=\bar{Y}\left(1+e_{0}\right)$, $\bar{x}_{1}=\bar{X}_{1}\left(1+e_{1}\right), \bar{x}_{2}=\bar{X}_{2}\left(1+e_{2}\right)$ such that $E\left(e_{0}\right)=E\left(e_{1}\right)=E\left(e_{2}\right)=0$ and $E\left(e_{0}^{2}\right)=\theta C_{y}^{2}, E\left(e_{1}^{2}\right)=\theta C_{x_{1}}^{2}$, $E\left(e_{2}^{2}\right)=\theta C_{x_{2}}^{2}, E\left(e_{0} e_{1}\right)=\theta \rho_{y x_{1}} C_{y} C_{x_{1}}, E\left(e_{0} e_{2}\right)=\theta \rho_{y x_{2}} C_{y} C_{x_{2}}$ and $E\left(e_{1} e_{2}\right)=\theta \rho_{x_{1} x_{2}} C_{x_{1}} C_{x_{2}}$.

Expressing the $\hat{\bar{Y}}_{7}$ in terms of $e_{i}^{\prime} s$, we get

$$
\begin{aligned}
\hat{\bar{Y}}_{7} & =\bar{Y}\left(1+e_{0}\right)\left(\frac{\bar{X}_{1} C_{x_{1}}+\beta_{2}\left(x_{1}\right)}{\bar{X}_{1}\left(1+e_{1}\right) C_{x_{1}}+\beta_{2}\left(x_{1}\right)}\right)\left(\frac{\bar{X}_{2}\left(1+e_{2}\right) C_{x_{2}}+\beta_{2}\left(x_{2}\right)}{\bar{X}_{2} C_{x_{2}}+\beta_{2}\left(x_{2}\right)}\right) \\
& =\bar{Y}\left(1+e_{0}\right)\left(\frac{\bar{X}_{1} C_{x_{1}}+\beta_{2}\left(x_{1}\right)}{\bar{X}_{1} C_{x_{1}}+\beta_{2}\left(x_{1}\right)+\bar{X}_{1} C_{x_{1}} e_{1}}\right)\left(\frac{\bar{X}_{2} C_{x_{2}}+\beta_{2}\left(x_{2}\right)+\bar{X}_{2} C_{x_{2}} e_{2}}{\bar{X}_{2} C_{x_{2}}+\beta_{2}\left(x_{2}\right)}\right) \\
& =\bar{Y}\left(1+e_{0}\right)\left(1+\lambda_{1} e_{1}\right)^{-1}\left(1+\lambda_{2} e_{2}\right) \\
& =\bar{Y}\left(1+e_{0}\right)\left(1-\lambda_{1} e_{1}+\lambda_{1}^{2} e_{1}^{2}\right)\left(1+\lambda_{2} e_{2}\right) \\
& =\bar{Y}\left(1+e_{0}\right)\left(1-\lambda_{1} e_{1}+\lambda_{1}^{2} e_{1}^{2}+\lambda_{1} \lambda_{2} e_{1} e_{2}+\lambda_{2} e_{2}\right) \\
\left(\hat{\bar{Y}}_{7}-\hat{Y}_{7}\right) & =\bar{Y}\left(1-\lambda_{1} e_{1}+\lambda_{1}^{2} e_{1}^{2}+\lambda_{1} \lambda_{2} e_{1} e_{2}+\lambda_{2} e_{2}+e_{0}-\lambda_{1} e_{0} e_{1}+\lambda_{2} e_{0} e_{2}\right) \\
& \bar{Y}\left(-\lambda_{1} e_{1}+\lambda_{1}^{2} e_{1}^{2}+\lambda_{2} e_{2}-\lambda_{1} \lambda_{2} e_{1} e_{2}+e_{0}-\lambda_{1} e_{0} e_{1}+\lambda_{2} e_{0} e_{2}\right) .
\end{aligned}
$$

Taking expectation of both sides of (2.3)

$$
E\left(\hat{\bar{Y}}_{7}-\bar{Y}\right)=\bar{Y} E\left(-\lambda_{1} e_{1}+\lambda_{1}^{2} e_{1}^{2}+\lambda_{2} e_{2}-\lambda_{1} \lambda_{2} e_{1} e_{2}+e_{0}-\lambda_{1} e_{0} e_{1}+\lambda_{2} e_{0} e_{2}\right) .
$$


Substituting the values of $E\left(e_{0}\right), E\left(e_{1}\right), E\left(e_{2}\right), E\left(e_{1}^{2}\right), E\left(e_{0} e_{1}\right), E\left(e_{0} e_{2}\right)$ and $E\left(e_{1} e_{2}\right)$ we get the bias of $\hat{\bar{Y}}_{7}$ as

$$
B\left(\hat{\bar{Y}}_{7}\right)=\theta \bar{Y}\left[\lambda_{1} C_{x_{1}}^{2}\left(\lambda_{1}-K_{y x_{1}}\right)+\lambda_{2} C_{x_{2}}^{2}\left(K_{y x_{2}}-\lambda_{1} K_{x_{1} x_{2}}\right)\right] .
$$

To find the mean squared error of the suggested estimator $\hat{\bar{Y}}_{7}$ up to first degree of approximation, squaring and taking expectation of (2.3)

$$
\begin{aligned}
E\left(\hat{\bar{Y}}_{7}-\bar{Y}\right)^{2} & =\bar{Y}^{2} E\left(e_{0}-\lambda_{1} e_{1}+\lambda_{2} e_{2}\right)^{2}, \\
\operatorname{MSE}\left(\hat{\bar{Y}}_{7}\right) & =\bar{Y}^{2} E\left(e_{0}^{2}+\lambda_{1}^{2} e_{1}^{2}+\lambda_{2}^{2} e_{2}^{2}-2 \lambda_{1} e_{0} e_{1}+2 \lambda_{2} e_{0} e_{2}-2 \lambda_{1} \lambda_{2} e_{1} e_{2}\right) .
\end{aligned}
$$

After substituting the values of $E\left(e_{0}^{2}\right), E\left(e_{1}^{2}\right), E\left(e_{2}^{2}\right), E\left(e_{0} e_{1}\right), E\left(e_{0} e_{2}\right)$ and $E\left(e_{1} e_{2}\right)$ we have mean squared error of $\hat{\bar{Y}}_{7}$ as

$$
\operatorname{MSE}\left(\hat{\bar{Y}}_{7}\right)=\theta \bar{Y}^{2}\left[C_{y}^{2}+\lambda_{1} C_{x_{1}}^{2}\left(\lambda_{1}-2 K_{y x_{1}}\right)+\lambda_{2} C_{x_{2}}^{2}\left\{\lambda_{2}+2\left(K_{y x_{2}}-\lambda_{1} K_{x_{1} x_{2}}\right)\right\}\right] .
$$

Similarly bias and mean squared error of $\hat{\bar{Y}}_{8}$ can be obtained as

$$
\begin{aligned}
B\left(\hat{\bar{Y}}_{8}\right) & =\theta \bar{Y}\left[\gamma_{1} C_{x_{1}}^{2}\left(\gamma_{1}-K_{y x_{1}}\right)+\gamma_{2} C_{x_{2}}^{2}\left(K_{y x_{2}}-\gamma_{1} K_{x_{1} x_{2}}\right)\right], \\
\operatorname{MSE}\left(\hat{\bar{Y}}_{8}\right) & =\theta \bar{Y}^{2}\left[C_{y}^{2}+\gamma_{1} C_{x_{1}}^{2}\left(\lambda_{1}-2 K_{y x_{1}}\right)+\gamma_{2} C_{x_{2}}^{2}\left\{\gamma_{2}+2\left(K_{y x_{2}}-\gamma_{1} K_{x_{1} x_{2}}\right)\right\}\right] .
\end{aligned}
$$

\section{Efficiency Comparison}

We know that the variance of sample mean $\bar{y}$ in simple random sampling without replacement(SRSW OR) is

$$
V(\bar{y})=\left(\frac{1}{n}-\frac{1}{N}\right) S_{y}^{2}
$$

From (1.9) to (1.16), (2.5), (2.7) and (3.1) we have

(i) $\operatorname{MSE}\left(\hat{\bar{Y}}_{7}\right)<\operatorname{MSE}(\bar{y})$ if

$$
K_{y x_{1}}>\frac{\lambda_{1}}{2} \quad \text { and } \quad K_{y x_{2}}>\left(\lambda_{1} K_{x_{1} x_{2}}-\frac{\lambda_{2}}{2}\right)
$$

(ii) $\operatorname{MSE}\left(\hat{\bar{Y}}_{7}\right)<\operatorname{MSE}\left(\bar{y}_{R}\right)$ if

$$
K_{y x_{1}}<\left(\frac{1+\lambda_{1}}{2}\right) \quad \text { and } \quad K_{y x_{2}}<\left(\lambda_{1} K_{x_{1} x_{2}}-\frac{\lambda_{2}}{2}\right)
$$

(iii) $\operatorname{MSE}\left(\hat{\bar{Y}}_{7}\right)<\operatorname{MSE}\left(\bar{y}_{P}\right)$ if

$$
K_{y x_{1}}>\left(\frac{\lambda_{1}}{2}-\lambda_{2} K_{x_{2} x_{1}}\right) \quad \text { and } \quad K_{y x_{2}}>-\left(\frac{1+\lambda_{2}}{2}\right)
$$


(iv) $\operatorname{MSE}\left(\hat{\bar{Y}}_{7}\right)<\operatorname{MSE}\left(\hat{\bar{Y}}_{1}\right)$ if

$$
K_{y x_{2}}<\left(\lambda_{1} K_{x_{1} x_{2}}-\frac{\lambda_{2}}{2}\right)
$$

(v) $\operatorname{MSE}\left(\hat{\bar{Y}}_{7}\right)<\operatorname{MSE}\left(\hat{\bar{Y}}_{2}\right)$ if

$$
K_{y x_{1}}>-\lambda_{2} K_{x_{2} x_{1}}+\frac{\lambda_{2}}{2}
$$

(vi) $\operatorname{MSE}\left(\hat{\bar{Y}}_{7}\right)<\operatorname{MSE}\left(\hat{\bar{Y}}_{3}\right)$ if

$$
\begin{aligned}
& \text { either } K_{y x_{1}}>\left(\frac{\gamma_{1}+\lambda_{1}}{2}\right) \text { if } \gamma_{1}<\lambda_{1} \text { and } K_{y x_{2}}<\left(\lambda_{1} K_{x_{1} x_{2}}-\frac{\lambda_{2}}{2}\right) \\
& \text { or } \quad K_{y x_{1}}<\left(\frac{\gamma_{1}+\lambda_{1}}{2}\right) \text { if } \gamma_{1}>\lambda_{1} \text { and } K_{y x_{2}}<\left(\lambda_{1} K_{x_{1} x_{2}}-\frac{\lambda_{2}}{2}\right)
\end{aligned}
$$

(vii) $\operatorname{MSE}\left(\hat{\bar{Y}}_{7}\right)<\operatorname{MSE}\left(\hat{\bar{Y}}_{4}\right)$ if

$$
\begin{aligned}
\text { either } & K_{y x_{2}}>-\left(\frac{\gamma_{2}+\lambda_{2}}{2}\right) \text { if } \gamma_{2}>\lambda_{2} \text { and } K_{y x_{1}}>\left(\frac{\lambda_{1}}{2}-\lambda_{2} K_{x_{2} x_{1}}\right) \\
\text { or } & K_{y x_{2}}<-\left(\frac{\gamma_{2}+\lambda_{2}}{2}\right) \text { if } \gamma_{2}<\lambda_{2} \text { and } K_{y x_{1}}>\left(\frac{\lambda_{1}}{2}-\lambda_{2} K_{x_{2} x_{1}}\right)
\end{aligned}
$$

(viii) $\operatorname{MSE}\left(\hat{\bar{Y}}_{7}\right)<\operatorname{MSE}\left(\hat{\bar{Y}}_{5}\right)$ if

$$
\begin{aligned}
& K_{y x_{1}}>-\left(\frac{1+\lambda_{2}}{2}\right) \text { if } \lambda_{2}<1 \text { and } K_{y x_{1}}>\left\{\frac{1+\lambda_{1}}{2}-\frac{K_{x_{2} x_{1}}\left(\lambda_{1} \lambda_{2}-1\right)}{\lambda_{1}-1}\right\} \\
& K_{y x_{1}}<-\left(\frac{1+\lambda_{2}}{2}\right) \text { if } \lambda_{2}>1 \text { and } K_{y x_{1}}>\left\{\frac{1+\lambda_{1}}{2}-\frac{K_{x_{2} x_{1}}\left(\lambda_{1} \lambda_{2}-1\right)}{\lambda_{1}-1}\right\}
\end{aligned}
$$

(ix) $\operatorname{MSE}\left(\hat{\bar{Y}}_{7}\right)<\operatorname{MSE}\left(\hat{\bar{Y}}_{6}\right)$ if one of the following conditions is satisfied

$$
\begin{aligned}
& K_{y x_{1}}<\left(\frac{\mu_{1}^{*}+\lambda_{1}}{2}\right) \text { if } \lambda_{1}<\mu_{1}^{*} \text { and } K_{y x_{2}}>\left\{\frac{K_{x_{1} x_{2}}\left(\lambda_{1} \lambda_{2}-\mu_{1}^{*} \mu_{2}^{*}\right)}{\lambda_{2}-\mu_{2}^{*}}-\frac{\lambda_{2}+\mu_{2}^{*}}{2}\right\} \text { if } \lambda_{2}<\mu_{2}^{*} \\
& K_{y x_{1}}<\left(\frac{\mu_{1}^{*}+\lambda_{1}}{2}\right) \text { if } \lambda_{1}<\mu_{1}^{*} \text { and } K_{y x_{2}}<\left\{\frac{K_{x_{1} x_{2}}\left(\lambda_{1} \lambda_{2}-\mu_{1}^{*} \mu_{2}^{*}\right)}{\lambda_{2}-\mu_{2}^{*}}-\frac{\lambda_{2}+\mu_{2}^{*}}{2}\right\} \text { if } \lambda_{2}>\mu_{2}^{*} \\
& K_{y x_{1}}>\left(\frac{\mu_{1}^{*}+\lambda_{1}}{2}\right) \text { if } \lambda_{1}>\mu_{1}^{*} \text { and } K_{y x_{2}}>\left\{\frac{K_{x_{1} x_{2}}\left(\lambda_{1} \lambda_{2}-\mu_{1}^{*} \mu_{2}^{*}\right)}{\lambda_{2}-\mu_{2}^{*}}-\frac{\lambda_{2}+\mu_{2}^{*}}{2}\right\} \text { if } \lambda_{2}<\mu_{2}^{*} \\
& K_{y x_{1}}>\left(\frac{\mu_{1}^{*}+\lambda_{1}}{2}\right) \text { if } \lambda_{1}>\mu_{1}^{*} \text { and } K_{y x_{2}}<\left\{\frac{K_{x_{1} x_{2}}\left(\lambda_{1} \lambda_{2}-\mu_{1}^{*} \mu_{2}^{*}\right)}{\lambda_{2}-\mu_{2}^{*}}-\frac{\lambda_{2}+\mu_{2}^{*}}{2}\right\} \text { if } \lambda_{2}>\mu_{2}^{*}
\end{aligned}
$$

(x) $\operatorname{MSE}\left(\hat{\bar{Y}}_{8}\right)<\operatorname{MSE}\left(\hat{\bar{Y}}_{7}\right)$ if of the following conditions is satisfied

$$
\frac{C_{x_{1}}^{2}}{C_{x_{2}}^{2}}<\frac{\gamma_{2} C_{x_{2}}^{2}\left\{\gamma_{2}+2\left(K_{y x_{2}}-\gamma_{1} K_{x_{1} x_{2}}\right)\right\}-\lambda_{2} C_{x_{2}}^{2}\left\{\lambda_{2}+2\left(K_{y x_{2}}-\lambda_{1} K_{x_{1} x_{2}}\right)\right\}}{\lambda_{1}-\gamma_{1}} .
$$


It is observed that the proposed estimators $\hat{\bar{Y}}_{j}(j=7,8)$ are biased. Bias is disadvantageous in many situations. Keeping this in view, a family of almost unbiased estimators is also proposed using Random Group technique envisaged by Quenouille (1956).

\section{A Family of Unbiased Estimators of Population Mean $\bar{Y}$ Using Random Group Method}

Suppose a simple random sample of size $n=g m$ is drawn without replacement and split at random into $g$ sub-samples, each of size $m$. Then Jack-knife type ratio-cum-product estimator for population mean $\bar{Y}$, using $\hat{\bar{Y}}_{7}$ is given as

$$
\hat{\bar{Y}}_{7 J}=\frac{1}{g} \sum_{j=1}^{g} \bar{y}_{j}^{\prime}\left(\frac{\bar{X}_{1} C_{x_{1}}+\beta_{2}\left(x_{1}\right)}{\bar{x}_{1 j}^{\prime} C_{x_{1}}+\beta_{2}\left(x_{1}\right)}\right)\left(\frac{\bar{x}_{2 j}^{\prime} C_{x_{2}}+\beta_{2}\left(x_{2}\right)}{\bar{X}_{2} C_{x_{2}}+\beta_{2}\left(x_{2}\right)}\right),
$$

where $\bar{y}_{j}^{\prime}=\left(n \bar{y}-m \bar{y}_{j}\right) /(n-m)$ and $\bar{x}_{i j}^{\prime}=\left(n \bar{x}_{i}-m \bar{x}_{i j}\right) /(n-m), i=1,2$; are the sample means based on a sample of $(n-m)$ units obtained by omitting the $j^{\text {th }}$ group and $\bar{y}_{j}$ and $\bar{x}_{i j}(i=1,2 ; j=1,2, \ldots, g)$ are the sample means based on the $j^{\text {th }}$ sub samples of size $m=n / g$.

The bias of $\hat{\bar{Y}}_{7 J}$, upto the first degree of approximation can be easily obtained as

$$
B\left(\hat{\bar{Y}}_{7 J}\right)=\frac{N-n+m}{N(n-m)} \bar{Y}\left[\lambda_{1} C_{x_{1}}^{2}\left(\lambda_{1}-K_{y x_{1}}\right)+\lambda_{2} C_{x_{2}}^{2}\left(K_{y x_{2}}-\lambda_{1} K_{x_{1} x_{2}}\right)\right] .
$$

From (2.4) and (4.2) we have

$$
\begin{aligned}
& \frac{B\left(\hat{\bar{Y}}_{7}\right)}{B\left(\hat{\bar{Y}}_{7 J}\right)}=\frac{(N-n)(n-m)}{n(N-n+m)} \quad \text { or } \quad B\left(\hat{\bar{Y}}_{7}\right)-\frac{(N-n)(n-m)}{n(N-n+m)} B\left(\hat{\bar{Y}}_{7 J}\right)=0 \\
& \Rightarrow \lambda^{*} B\left(\hat{\bar{Y}}_{7}\right)-\delta^{*} \lambda^{*} B\left(\hat{\bar{Y}}_{7 J}\right)=0
\end{aligned}
$$

for any scalar $\lambda^{*}$, where

$$
\delta^{*}=\frac{(N-n)(n-m)}{n(N-n+m)} .
$$

From (4.4), we have

$$
\begin{aligned}
& \lambda^{*} E\left(\hat{\bar{Y}}_{7}-\bar{Y}\right)-\delta^{*} \lambda^{*} E\left(\hat{\bar{Y}}_{7 J}-\bar{Y}\right)=0 \quad \text { or } \quad \lambda^{*} E\left(\hat{\bar{Y}}_{7}-\bar{y}\right)-\delta^{*} \lambda^{*} E\left(\hat{\bar{Y}}_{7 J}-\bar{y}\right)=0 \quad \text { or } \\
& E\left[\lambda^{*} \hat{\bar{Y}}_{7}-\lambda^{*} \delta^{*} \hat{\bar{Y}}_{7 J}-\bar{y}\left\{\lambda^{*}\left(1-\delta^{*}\right)-1\right\}\right]=\bar{Y} .
\end{aligned}
$$

Thus we get a general family of almost unbiased ratio-cum-product estimators of $\bar{Y}$ as

$$
\hat{\bar{Y}}_{7 u}=\left[\bar{y}\left\{1-\lambda^{*}\left(1-\delta^{*}\right)\right\}+\lambda^{*} \hat{\bar{Y}}_{7}-\lambda^{*} \delta^{*} \hat{\bar{Y}}_{7 J}\right] .
$$

Remark 1. For $\lambda^{*}=0, \hat{\bar{Y}}_{7 u}$ yields the usual unbiased estimator $\bar{y}$ while $\lambda^{*}=\left(1-\delta^{*}\right)^{-1}$, gives an almost unbiased estimator for $\bar{Y}$ as

$$
\begin{aligned}
\hat{\bar{Y}}_{7 u}^{*}= & \frac{(N-n+m)}{N} g \bar{y}\left(\frac{\bar{X}_{1} C_{x_{1}}+\beta_{2}\left(x_{1}\right)}{\bar{x}_{1} C_{x_{1}}+\beta_{2}\left(x_{1}\right)}\right)\left(\frac{\bar{x}_{2} C_{x_{2}}+\beta_{2}\left(x_{2}\right)}{\bar{X}_{2} C_{x_{2}}+\beta_{2}\left(x_{2}\right)}\right) \\
& -\frac{(N-n)(g-1)}{N g} \sum_{j=1}^{g} \bar{y}_{j}^{\prime}\left(\frac{\bar{X}_{1} C_{x_{1}}+\beta_{2}\left(x_{1}\right)}{\bar{x}_{1 j}^{\prime} C_{x_{1}}+\beta_{2}\left(x_{1}\right)}\right)\left(\frac{\bar{x}_{2 j}^{\prime} C_{x_{2}}+\beta_{2}\left(x_{2}\right)}{\bar{X}_{2} C_{x_{2}}+\beta_{2}\left(x_{2}\right)}\right) .
\end{aligned}
$$


Which is Jack-knifed version of the proposed estimator $\hat{\bar{Y}}_{7}$.

Different suites values of $\lambda^{*}$ provides many almost unbiased estimators in (4.6).

\section{An Optimum Estimator In Family $\hat{\bar{Y}}_{7 u}$}

The family of almost unbiased estimator $\hat{\bar{Y}}_{7 u}$ at (4.6) can be expressed as

$$
\hat{\bar{Y}}_{7 u}=\bar{y}-\lambda^{*} \bar{y}_{1},
$$

where $\bar{y}_{1}=\left[\left(1-\delta^{*}\right) \bar{y}-\bar{y}_{2}\right]$ and $\bar{y}_{2}=\hat{\bar{Y}}_{7}-\delta^{*} \hat{\bar{Y}}_{7 J}$.

The variance of $\hat{\bar{Y}}_{7 u}$ is given by

$$
V\left(\hat{\bar{Y}}_{7 u}\right)=V(\bar{y})+\lambda^{* 2} V\left(\bar{y}_{1}\right)-2 \lambda^{*} \operatorname{Cov}\left(\bar{y}, \bar{y}_{1}\right)
$$

which is minimized for

$$
\lambda^{*}=\operatorname{Cov}\left(\bar{y}, \bar{y}_{1}\right) / V\left(\bar{y}_{1}\right)
$$

Substitution of (5.3) in (5.2) yields minimum variance of $\hat{\bar{Y}}_{7 u}$ as

$$
\min . V\left(\hat{\bar{Y}}_{7 u}\right)=V(\bar{y})-\frac{\left\{\operatorname{Cov}\left(\bar{y}, \bar{y}_{1}\right)\right\}^{2}}{V\left(\bar{y}_{1}\right)}=V(\bar{y})\left(1-\rho_{01}^{2}\right),
$$

where $\rho_{01}$ is the correlation coefficient between $\bar{y}$ and $\bar{y}_{1}$.

From (5.4) it is clear that min. $V\left(\hat{\bar{Y}}_{7 u}\right)<V(\bar{y})$.

To obtain the explicit expression of the variance of $\hat{\bar{Y}}_{7 u}$, we write the following results upto terms of order $n^{-1}$, as

$$
\operatorname{MSE}\left(\hat{\bar{Y}}_{7 J}\right)=\operatorname{Cov}\left(\hat{\bar{Y}}_{7}, \hat{\bar{Y}}_{7 J}\right)=\operatorname{MSE}\left(\hat{\bar{Y}}_{7}\right)
$$

and

$$
\operatorname{Cov}\left(\bar{y}, \hat{\bar{Y}}_{7}\right)=\operatorname{Cov}\left(\bar{y}, \hat{\bar{Y}}_{7 J}\right)=\theta \bar{Y}^{2}\left[C_{y}^{2}-\lambda_{1} \rho_{y x_{1}} C_{y} C_{x_{1}}+\lambda_{2} \rho_{y x_{2}} C_{y} C_{x_{2}}\right],
$$

where $\operatorname{MSE}\left(\hat{\bar{Y}}_{7}\right)$ is given by (2.5).

Using (2.5), (3.1) and (5.6) in (5.2), the variance of $\hat{\bar{Y}}_{7 u}$ upto the terms of order $n^{-1}$ is given as

$$
\begin{aligned}
V\left(\hat{\bar{Y}}_{7 u}\right)= & \theta \bar{Y}^{2}\left[C_{y}^{2}+\lambda^{* 2}\left(1-\delta^{*}\right)^{2}\left(\lambda_{1}^{2} C_{x_{1}}^{2}+\lambda_{2}^{2} C_{x_{2}}^{2}-2 \rho_{x_{1} x_{2}} C_{x_{1}} C_{x_{2}} \lambda_{1} \lambda_{2}\right)\right. \\
& \left.-2 \lambda^{*}\left(1-\delta^{*}\right)\left(\lambda_{1} \rho_{y x_{1}} C_{y} C_{x_{1}}-\lambda_{2} \rho_{y x_{2}} C_{y} C_{x_{2}}\right)\right],
\end{aligned}
$$

which is minimized for

$$
\lambda^{*}=\frac{\lambda_{1} \rho_{y x_{1}} C_{y} C_{x_{1}}-\lambda_{2} \rho_{y x_{2}} C_{y} C_{x_{2}}}{\left(1-\delta^{*}\right)\left(\lambda_{1}^{2} C_{x_{1}}^{2}+\lambda_{2}^{2} C_{x_{2}}^{2}-2 \lambda_{1} \lambda_{2} \rho_{x_{1} x_{2}} C_{x_{1}} C_{x_{2}}\right)}=\lambda_{o p t}^{*} .
$$

Substitution of the value of $\lambda_{o p t}^{*}$ in $\hat{\bar{Y}}_{7 u}$ yields the optimum estimator $\hat{\bar{Y}}_{7 u(o p t)}$ (say). Thus the resulting minimum variance of $\hat{\bar{Y}}_{7 u}$ is given by

$$
\min . V\left(\hat{\bar{Y}}_{7 u}\right)=\theta \bar{Y}^{2} C_{y}^{2}\left[1-\frac{\left(\lambda_{1} \rho_{y x_{1}} C_{x_{1}}-\lambda_{2} \rho_{y x_{2}} C_{x_{2}}\right)^{2}}{\lambda_{1}^{2} C_{x_{1}}^{2}+\lambda_{2}^{2} C_{x_{2}}^{2}-2 \lambda_{1} \lambda_{2} \rho_{x_{1} x_{2}} C_{x_{1}} C_{x_{2}}}\right]=V\left(\hat{\bar{Y}}_{7 u(o p t)}\right)
$$


The optimum value $\lambda_{o p t}^{*}$ of $\lambda^{*}$ can be obtained quite accurately through past data or experience.

Adopting the similar procedure, using proposed estimator $\hat{\bar{Y}}_{8}$, we can obtain an almost unbiased family of estimators $\hat{\bar{Y}}_{8 u}$.

Further the variance of the proposed almost unbiased family of estimators $\hat{\bar{Y}}_{8 u}$ to the first degree of approximation is given by

$$
\begin{aligned}
V\left(\hat{\bar{Y}}_{8 u}\right)= & \theta \bar{Y}^{2}\left[C_{y}^{2}+\lambda^{* 2}\left(1-\delta^{*}\right)^{2}\left(\gamma_{1}^{2} C_{x_{1}}^{2}+\gamma_{2}^{2} C_{x_{2}}^{2}-2 \rho_{x_{1} x_{2}} C_{x_{1}} C_{x_{2}} \gamma_{1} \gamma_{2}\right)\right. \\
& \left.-2 \gamma^{*}\left(1-\delta^{*}\right)\left(\gamma_{1} \rho_{y x_{1}} C_{y} C_{x_{1}}-\gamma_{2} \rho_{y x_{2}} C_{y} C_{x_{2}}\right)\right] \\
\lambda^{*}= & \frac{\gamma_{1} \rho_{y x_{1}} C_{y} C_{x_{1}}-\gamma_{2} \rho_{y x_{2}} C_{y} C_{x_{2}}}{\left(1-\delta^{*}\right)\left(\gamma_{1}^{2} C_{x_{1}}^{2}+\gamma_{2}^{2} C_{x_{2}}^{2}-2 \gamma_{1} \gamma_{2} \rho_{x_{1} x_{2}} C_{x_{1}} C_{x_{2}}\right)}=\gamma_{o p t}^{*}
\end{aligned}
$$

and resulting $\min . V\left(\hat{\bar{Y}}_{8 u}\right)$ is obtained as

$$
\min . V\left(\hat{\bar{Y}}_{8 u}\right)=\theta \bar{Y}^{2} C_{y}^{2}\left[1-\frac{\left(\gamma_{1} \rho_{y x_{1}} C_{x_{1}}-\gamma_{2} \rho_{y x_{2}} C_{x_{2}}\right)^{2}}{\gamma_{1}^{2} C_{x_{1}}^{2}+\gamma_{2}^{2} C_{x_{2}}^{2}-2 \gamma_{1} \gamma_{2} \rho_{x_{1} x_{2}} C_{x_{1}} C_{x_{2}}}\right]=V\left(\hat{\bar{Y}}_{8 u(o p t)}\right) \text {. }
$$

\section{Empirical Study}

To observe the relative performance of different estimators of $\bar{Y}$, a natural population data sets is being considered

\section{- Population [Source: Steel and Torrie (1960, p.282)]}

$y$ : Log of leaf burn in sec.,

$x_{1}$ : Potassiam percentage,

$x_{2}$ : Clorine percentage.

The required population parameters are

$$
\begin{aligned}
& \bar{Y}=0.6860, \quad C_{y}=0.4803, \quad \rho_{y x_{1}}=0.1794, \quad N=30, \\
& \bar{X}_{1}=4.6537, \quad C_{x_{1}}=0.2295, \quad \rho_{y x_{2}}=-0.4996, \quad \beta_{2}\left(x_{1}\right)=1.56, \quad n=6 \text {, } \\
& \bar{X}_{2}=0.8077, \quad C_{x_{2}}=0.7493, \quad \rho_{x_{1} x_{2}}=0.4074, \quad \beta_{2}\left(x_{2}\right)=1.40 \text {. }
\end{aligned}
$$

To see the performance of the various estimators in comparison to $\bar{y}$, we calculate the percent relative efficiency of all estimators with respect to $\bar{y}$ which is the ratio of the variance of $\bar{y}$ to the mean squared error of the estimator multiplied by 100 . The Percent relative efficiency $(\%)$ of the estimators $\bar{y}, \bar{y}_{R}, \bar{y}_{P}, \hat{\bar{Y}}_{1}, \hat{\bar{Y}}_{2}, \hat{\bar{Y}}_{3}, \hat{\bar{Y}}_{4}, \hat{\bar{Y}}_{5}, \hat{\bar{Y}}_{6}, \hat{\bar{Y}}_{7}, \hat{\bar{Y}}_{7}^{(o p t)}, \hat{\bar{Y}}_{8}$ and $\hat{\bar{Y}}_{8}^{(o p t)}$ have been computed and presented in Table 1. Formulae for percent relative efficiencies of different estimators are given below:

$$
\begin{aligned}
& \operatorname{PRE}\left(\bar{y}_{R}, \bar{y}\right)=\frac{V(\bar{y})}{\operatorname{MSE}\left(\bar{y}_{R}\right)}=\frac{C_{y}^{2}}{C_{y}^{2}+C_{x_{1}}^{2}-2 \rho_{y x_{1}} C_{y} C_{x_{1}}} \times 100 \\
& \operatorname{PRE}\left(\bar{y}_{P}, \bar{y}\right)=\frac{V(\bar{y})}{\operatorname{MSE}\left(\bar{y}_{P}\right)} \times 100=\frac{V(\bar{y})}{C_{y}^{2}+C_{x_{1}}^{2}+2 \rho_{y x_{1}} C_{y} C_{x_{1}}} \times 100 \\
& \operatorname{PRE}\left(\hat{\bar{Y}}_{1}, \bar{y}\right)=\frac{V(\bar{y})}{\operatorname{MSE}\left(\hat{\bar{Y}}_{1}\right)} \times 100=\frac{C_{y}^{2}}{C_{y}^{2}+\lambda_{1} C_{x_{1}}^{2}-2 \rho_{y x_{1}} \lambda_{1} C_{y} C_{x_{1}}} \times 100
\end{aligned}
$$


Table 1: Percent relative efficiencies of different estimators of with respect to

\begin{tabular}{cccccccc}
\hline \hline Estimators & $\bar{y}$ & $\bar{y}_{R}$ & $\bar{y}_{P}$ & $\hat{\bar{Y}}_{1}$ & $\hat{\bar{Y}}_{2}$ & $\hat{\bar{Y}}_{3}$ & $\hat{\hat{Y}}_{4}$ \\
\hline PREs & 100.00 & 94.62 & 53.33 & 97.21 & 58.74 & 95.39 & 106.06 \\
\hline Estimators & $\hat{\bar{Y}}_{5}$ & $\hat{\bar{Y}}_{6}$ & $\hat{\bar{Y}}_{7}$ & $\hat{\bar{Y}}_{7}^{(\text {(opt })}$ & $\hat{\bar{Y}}_{8}$ & $\hat{Y}_{8}^{(\text {(opt })}$ & \\
\hline PREs & 75.50 & 142.17 & $\mathbf{1 5 5 . 1 0}$ & $\mathbf{1 6 9 . 8 1}$ & $\mathbf{1 5 6 . 9 6}$ & $\mathbf{1 6 5 . 1 4}$ & \\
\hline \hline
\end{tabular}

$$
\begin{aligned}
\operatorname{PRE}\left(\hat{\bar{Y}}_{2}, \bar{y}\right) & =\frac{V(\bar{y})}{\operatorname{MSE}\left(\hat{\bar{Y}}_{2}\right)} \times 100=\frac{C_{y}^{2}}{C_{y}^{2}+\lambda_{2} C_{x_{2}}^{2}+2 \rho_{y x_{2}} \lambda_{2} C_{y} C_{x_{2}}} \times 100 \\
\operatorname{PRE}\left(\hat{\bar{Y}}_{3}, \bar{y}\right) & =\frac{V(\bar{y})}{\operatorname{MSE}\left(\hat{\bar{Y}}_{3}\right)} \times 100=\frac{C_{y}^{2}}{C_{y}^{2}+\gamma_{1}^{2} C_{x_{1}}^{2}-2 \rho_{y x_{1}} \gamma_{1} C_{y} C_{x_{1}}} \times 100 \\
\operatorname{PRE}\left(\hat{\bar{Y}}_{4}, \bar{y}\right) & =\frac{V(\bar{y})}{\operatorname{MSE}\left(\hat{\bar{Y}}_{4}\right)} \times 100=\frac{C_{y}^{2}}{C_{y}^{2}+\gamma_{2}^{2} C_{x_{2}}^{2}+2 \rho_{y x_{2}} \gamma_{2} C_{y} C_{x_{2}}} \times 100 \\
\operatorname{PRE}\left(\hat{\bar{Y}}_{5}, \bar{y}\right) & =\frac{V(\bar{y})}{\operatorname{MSE}\left(\hat{\bar{Y}}_{5}\right)} \times 100=\frac{C_{y}^{2}}{C_{y}^{2}+C_{x_{1}}^{2}\left(1-2 K_{y x_{1}}\right)+C_{x_{2}}^{2}\left\{1+2\left(K_{y x_{2}}-K_{x_{1} x_{2}}\right)\right\}} \times 100 \\
\operatorname{PRE}\left(\hat{\bar{Y}}_{6}, \bar{y}\right) & =\frac{V(\bar{y})}{\operatorname{MSE}\left(\hat{\bar{Y}}_{6}\right)} \times 100=\frac{C_{y}^{2}}{C_{y}^{2}+\mu_{1}^{*} C_{x_{1}}^{2}\left(\mu_{1}^{*}-2 K_{y x_{1}}\right)+\mu_{2}^{*} C_{x_{2}}^{2}\left\{\mu_{1}^{*}+2\left(K_{y x_{2}}-\mu_{1}^{*} K_{x_{1} x_{2}}\right)\right\}} \times 100 \\
\operatorname{PRE}\left(\hat{\bar{Y}}_{7}^{(o p t)}, \bar{y}\right) & =\frac{V\left(\hat{\bar{Y}}_{7}, \bar{y}\right)}{\operatorname{MSE}\left(\hat{\bar{Y}}_{7}^{(o p t)}\right)} \times 100=\frac{C_{y}^{2}}{C_{y}^{2}+\lambda_{1} C_{x_{1}}^{2}\left(\lambda_{1}-2 K_{y x_{1}}\right)+\lambda_{2} C_{x_{2}}^{2}\left\{\lambda_{2}+2\left(K_{y x_{2}}-\lambda_{1} K_{x_{1} x_{2}}\right)\right\}} \times 100 \\
\operatorname{PRE}\left(\hat{\bar{Y}}_{8}, \bar{y}\right) & =\frac{V(\bar{y})}{\operatorname{MSE}\left(\hat{\bar{Y}}_{8}\right)} \times 100=\frac{1-\frac{\left(\lambda_{1} \rho_{y x_{1}} C_{x_{1}}-\lambda_{2} \rho_{y x_{2}} C_{x_{2}}\right)^{2}}{\lambda_{1}^{2} C_{x_{1}}^{2}+\lambda_{2}^{2} C_{x_{2}}^{2}-2 \lambda_{1} \lambda_{2} \rho_{x_{1} x_{2}} C_{x_{1}} C_{x_{2}}}}{C_{y}^{2}+\gamma_{1} C_{x_{1}}^{2}\left(\gamma_{1}-2 K_{y x_{1}}\right)+\gamma_{2} C_{x_{2}}^{2}\left\{\gamma_{2}+2\left(K_{y x_{2}}-\gamma_{1} K_{x_{1} x_{2}}\right)\right\}} \times 100 \\
\operatorname{PRE}\left(\hat{\bar{Y}}_{8}^{(o p t)}, \bar{y}\right) & =\frac{V(\bar{y})}{\operatorname{MSE}\left(\hat{\bar{Y}}_{8}^{(o p t)}\right)} \times 100=\frac{1}{1-\frac{\left(\gamma_{1} \rho_{y x_{1}} C_{x_{1}}-\gamma_{2} \rho_{y x_{2}} C_{x_{2}}\right)^{2}}{\gamma_{1}^{2} C_{x_{1}}^{2}+\gamma_{2}^{2} C_{x_{2}}^{2}-2 \gamma_{1} \gamma_{2} \rho_{x_{1} x_{2}} C_{x_{1}} C_{x_{2}}}} \times 100 . \\
& C_{y}^{2}
\end{aligned}
$$

Table 1 shows that the suggested estimators $\hat{\bar{Y}}_{7}\left(\right.$ or $\hat{\bar{Y}}_{7}^{(o p t)}$ ) and $\hat{\bar{Y}}_{8}\left(\right.$ or $\hat{\bar{Y}}_{8}^{(o p t)}$ ) with $\lambda^{*}=\lambda_{\text {opt })}^{*}$ and $\alpha^{*}=$ $\alpha_{(o p t)}^{*}$ are more efficient than usual unbiased estimator $\bar{y}$, ratio estimator $\bar{y}_{r}$, product estimator $\bar{y}_{P}$, ratiocum-product estimators suggested by Singh (1967) and Singh and Tailor (2005) with considerable gain in efficiency. Thus, if coefficient of variation $\left(C_{x_{1}}\right.$ and $\left.C_{x_{2}}\right)$ and coefficient of kurtosis $\left(\beta_{2}\left(x_{1}\right)\right.$ and $\left.\beta_{2}\left(x_{2}\right)\right)$ are known of auxiliary variates $x_{1}$ and $x_{2}$, both estimators are recommended for use in practice.

\section{Acknowledgements}

Authors are thankful to referee for his valuable suggestions regarding the improvement of the paper. 


\section{References}

Cochran, W. G. (1940). The estimation of the yields of the cereal experiments by sampling for the ratio of grain to total produce, The Journal of Agricultural Science, 30, 262-275.

Murthy, M. N. (1967). Sampling Theory and Methods, Statistical Publishing Society, Calcultta.

Quenouille, M. H. (1956). Notes on bias in estimation, Biometrika, 43, 353-360.

Robson, D. S. (1957). Application of multivariate polykays to the theory of unbiased ratio-type estimation, Journal of the American Statistical Association, 52, 511-522.

Singh, H. P. and Tailor, R. (2005). Estimation of finite population mean using known correlation coefficient between auxiliary characters, Statistica, 65, 407-418.

Singh, M. P. (1967). Ratio-cum-product method of estimation, Metrika, 12, 34-42.

Sisodia, B. V. S. and Dwivedi, V. K. (1981). A modified ratio estimator using coefficient of variation of auxiliary variable, Journal Indian Society Agricultural Statistics, 33, 13-18.

Upadhyaya, L. N. and Singh, H. P. (1999). Use of transformed auxiliary variable in estimating the finite population mean, Biometrical Journal, 41, 627-636. 